\title{
Extração de características em imagens para reconhecimento de padrões
}

\author{
Robson Brito Santos ${ }^{1}$, César Alberto da Silva ${ }^{1,2}$, Linnyer Beatrys Ruiz Aylon ${ }^{2,3}$ \\ ${ }^{1}$ Instituto Federal de São Paulo - Câmpus Presidente Epitácio (IFSP) \\ CEP 19470-000 - Presidente Epitácio - SP - Brasil \\ ${ }^{2}$ Departamento de Engenharia Elétrica - Universidade Federal de Minas Gerais (UFMG) \\ CEP 31270-901 - Belo Horizonte - MG - Brasil \\ ${ }^{3}$ Departamento de Computação - Universidade Estadual de Maringá (UEM) \\ CEP 87020-900 - Maringá - PR - Brasil \\ robson.santos@aluno.ifsp.edu.br, cesar@ifsp.edu.br, lbruiz@uem.br
}

\begin{abstract}
This paper presents an investigation of the segmentation and extraction algorithms of image characteristics to allow object recognition. A set of images was obtained from a ranarium and used in the experiment. The experiment consisted in using a segmentation algorithm and feature extraction and carrying out a comparative study of the results of three classification algorithms. The Random Forest algorithm obtained a higher index of correctly classified instances.
\end{abstract}

Resumo. Este artigo apresenta uma investigação dos algoritmos de segmentação e extração de características de imagens de modo a permitir o reconhecimento objetos. Um conjunto de imagens foram obtidas de um ranário e utilizadas no experimento. $O$ experimento consistiu em utilizar um algoritmo para segmentação e extração de características e realizar um estudo comparativo dos resultados de três algoritmos de classificação. $O$ algoritmo Random Forest obteve maior índice de instâncias classificadas corretamente.

\section{Introdução}

A atividade de reconhecimento de objetos ou animais em imagens é parte das aplicações de Visão Computacional. De acordo com Gonzalez e Woods (2002), um sistema de Visão Computacional deve possuir a habilidade de extrair somente as informações importantes, dentre as quais podem existir algumas informações irrelevantes. O sistema deve ser capaz de fazer inferências a partir de informações incompletas e conseguir identificar um padrão em uma imagem com a maior independência possível em relação a fatores como mudanças de posição, tamanho, orientação e variações no ponto de vista (transformações geométricas) (Silva et al., 2012).

O reconhecimento de objetos em imagens pode ser empregado em diversas áreas, principalmente nas áreas de inteligência artificial e de recuperação de informações. Como exemplo de aplicações pode-se citar a busca de imagem baseada em conteúdo, mineração de dados em vídeo, identificação de objetos para robôs móveis, entre outras (Silva et al., 2012).

Diferentes áreas do conhecimento estão relacionadas à visão computacional. Enquanto a biologia estuda as atividades neurais envolvidas na formação e percepção das imagens pelos seres vivos, a visão computacional analisa todos os processos e algoritmos usados pelas máquinas para reconhecer o que os humanos reconhecem 
(Shapiro, 2013). Para esse reconhecimento é necessário extrair informações das imagens e para facilitar a extração dessas informações, a visão computacional utiliza-se de um conjunto de técnicas de aprendizado de máquina (Lee et al., 2015).

Reconhecer padrões em imagem é uma tarefa que depende da quantidade de características que se conhece de cada objeto. Portanto, a extração das informações é uma etapa crucial no processo de reconhecimento (Khodaskar e Ladhake, 2014).

A segmentação é uma etapa importante do processamento de imagem. Essa etapa consiste na partição da imagem em regiões de interesse a fim de extrair características dessas regiões. Entretanto, para obter um reconhecimento de padrões eficiente é importante ter uma segmentação precisa e robusta (Chaou et al., 2015).

Este trabalho está inserido no contexto do Instituto Nacional de Ciência e Tecnologia de Sistemas Nano e Microeletrônicos, o INCT NAMITEC, que é formado por 137 pesquisadores de 32 instituições de pesquisa no Brasil e que está nas 05 regiões geográficas do Brasil. O objetivo deste trabalho é comparar os resultados dos algoritmos J48, Random Tree e Random Forest na classificação de imagens de anuros.

\section{Materiais e Métodos}

Esta pesquisa pode ser considerada do tipo exploratória porque envolve o levantamento bibliográfico, realização de experimentos práticos e análise dos resultados para a compreensão do problema estudado. Além disso, visa produzir conhecimentos, adquirir experiência, contribuir para a discussão da área ou fazer avançar o debate acerca dos assuntos abordados.

Inicialmente, foi realizada a revisão bibliográfica e em seguida foi escolhido o software para explorar os algoritmos de segmentação e extração de características. Os materiais utilizados foram um computador com acesso a Internet e os softwares Pynovisão para segmentação e extração das características das imagens e o Weka para a classificação das características.

\section{Pynovisão}

A segmentação agrupa os pixels de maneira lógica para simplificar o problema da extração de informações a partir de imagens (Santos Ferreira et al., 2017). Os segmentos devem conter pixels que são semelhantes em cor e textura (Achanta et al., 2012). Neste trabalho, foi efetuada a segmentação por meio dos algoritmos do Pynovisão, como mostra a Figura 1.

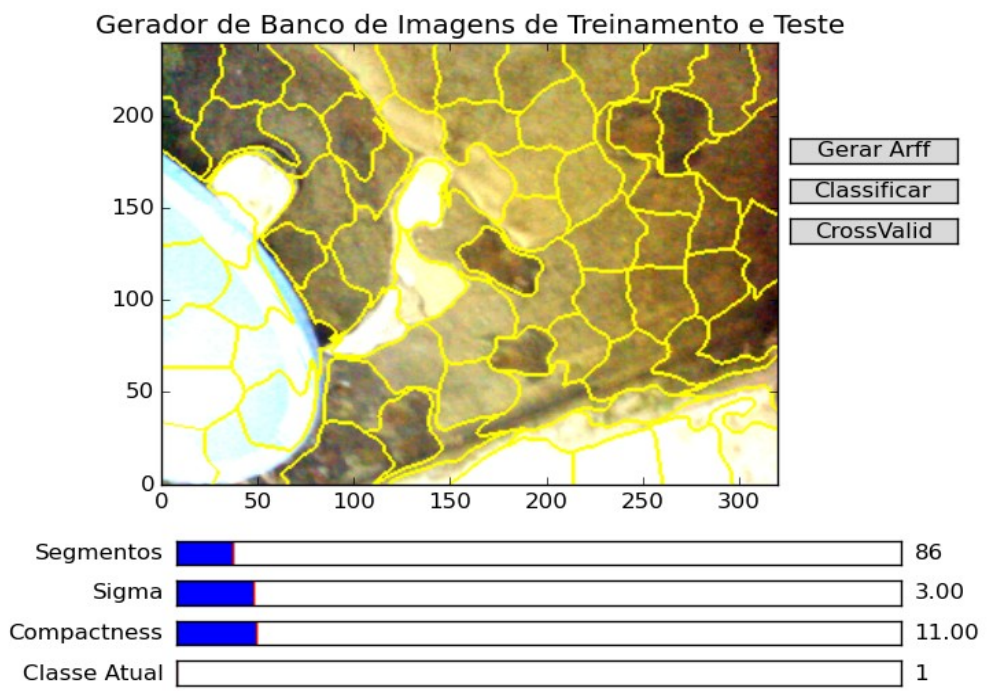

Figura 1: Imagem do ranario após a execução do algoritmo SLIC do Pynovisão. 
Achanta et al. (2012) explicam que os algoritmos capturam as redundâncias na imagem e reduz significativamente a complexidade das tarefas subsequentes do processamento de imagens.

Na segmentação das imagens das rãs foi utilizado o Algoritmo Simple Linear Iterative Clustering (SLIC). A abordagem utilizada por este algoritmo é gerar SuperPixels agrupando pixels com base em sua semelhança de cor e proximidade do plano da imagem. Isto é feito em cinco dimensões (5D) labxy, onde lab é o vetor de cor do pixel no espaço de cor CIELAB, e xy é a posição de cada pixel (Achanta et al., 2012).

Segundo Achanta et al. (2012), o SLIC é um algoritmo que tem apresentado maior eficiência quando comparado com algoritmos similares de segmentação. Entretanto, através dos testes realizados, percebeu-se que o algoritmo pode tornar-se menos significativo, afetando as estatísticas de aprendizagem de máquina, quando o banco de imagens é inferior a 60 figuras, quando há mudanças nos parâmetros e quando existem poucas classes.

Após a segmentação da imagem, as características de cada segmento são armazenadas em um arquivo que posteriormente é utilizado pelos algoritmos de classificação. Os algoritmos de classificação realizam o treinamento e definem um padrão para classificação dos dados.

\section{Resultados}

As imagens utilizadas no experimento foram obtidas no ranário da Universidade do Oeste Paulista - UNOESTE. Foram coletadas 41 imagens do ranário e foram utilizados três algoritmos de classificação, J48, Random Forest e Random Tree.

Inicialmente foi utilizado o algoritmo SLIC para segmentação das imagens e para cada segmento foi extraído um conjunto de características contendo 257 atributos. $\mathrm{O}$ conjunto de característica é formado por 7 atributos de Momentos de $\mathrm{Hu}, 36$ atributos calculados de cada um dos canais RGB, HSV e CIELab color spaces, 36 atributos extraídos da textura baseada em Gray-Level Co-occurrence Matrix (GLCM), 128 atributos extraídos do Histogram of Oriented Gradients (HOG), 18 atributos baseados no Local Binary Patterns (LBP) e 32 atributos extraídos do Gabor Filter.

O conjunto de característica de um segmento é chamado de uma instância. Com uso da ferramenta Weka, as instâncias de todas as imagens segmentadas foram utilizadas nos algoritmos de classificação, sendo 90\% das instâncias utilizadas para treinamento e $10 \%$ para os testes de classificação. A divisão das instâncias foi feita utilizando o algoritmo Fisher-Yates shuffle sem considerar a divisão das classes. Três algoritmos foram avaliados na classificação das instâncias, J48, Random Tree e Random Forest, a fim de encontrar um padrão entre as instâncias.

A Tabela 1 mostra os resultados dos três algoritmos de classificação em relação ao Erro Médio Absoluto, Raiz do Erro Quadrático Médio e a Taxa de Instâncias Classificadas Corretamente.

Tabela 1: Comparativo entre os algoritmos J48, Random Tree e Random Forest.

\begin{tabular}{|l|c|c|c|}
\hline & J48 & Random Tree & Random Forest \\
\hline Erro Médio Absoluto & 0,1062 & 0,0972 & 0,1063 \\
\hline Raiz do Erro Quadrático Médio & 0,3172 & 0,3117 & 0,2251 \\
\hline Instâncias Classificadas Corretamente & $89,47 \%$ & $90,28 \%$ & $93,52 \%$ \\
\hline
\end{tabular}




\section{Conclusão}

Este trabalho está em andamento e apresenta resultados parciais do uso do algoritmo SLIC para segmentação de imagens e extração de características utilizando o software Pynovisão. No experimento realizado foram comparados os resultados de três algoritmos de classificação, onde o algoritmo Random Forest obteve maior índice de instâncias classificadas corretamente. Experimentos adicionais serão realizados com mais imagens e outros parâmetros de configuração do algoritmo de segmentação.

\section{References}

Achanta, R., Shaji, A., Smith, K., Lucchi, A., Fua, P. and Süsstrunk, S. (2012) "Slic superpixels compared to state-of-the-art superpixel methods", In: IEEE Transactions on Pattern Analysis and Machine Intelligence, v. 34, n. 11, p. 2274-2282.

Chaou, A. K., Mekhaldi, A. and Teguar, M. (2015) "Elaboration of novel image processing algorithm for arcing discharges recognition on hv polluted insulator model”, In: IEEE Transactions on Dielectrics and Electrical Insulation, v. 22, n. 2, p. 990-999.

Santos Ferreira, A., Freitas, D. M., Silva, G. G., Pistori, H., and Folhes, M. T. (2017). Weed detection in soybean crops using ConvNets. Computers and Electronics in Agriculture, 143, 314-324.

Gonzalez, R. C. and Woods, R. E. (2002) "Digital image processing", New Jersey: Prentice-Hall, Inc..

Khodaskar, A. A. and Ladhake, S. A. (2014) "Pattern recognition: Advanced development, techniques and application for image retrieval", In: Communication and Network Technologies (ICCNT), 2014 International Conference on, p. 74-78.

Lee, W. P., Osman, M. A., Talib, A. Z., Yahya, K., Burie, J. C., Ogier, J. M. and Mennesson, J. (2015) "Recognition of fish based on generalized color fourier descriptor", In: Science and Information Conference (SAI), p. 680-686.

Shapiro, L. G. (2013) "Image analysis for biomedical and healthcare applications", In: Proceedings of the 1st ACM International Workshop on Multimedia Indexing and Information Retrieval for Healthcare, Barcelona, Spain, MIIRH '13, p. 1-2, New York, NY, USA. ACM.

Silva, F.A., Artero, A. O., Paiva, M. S. V. and Barbosa, R. L. (2012) "Uma Metodologia para Detectar e Reconhecer Placas de Sinalização de Trânsito", In: VIII Workshop de Visão Computacional - WVC 2012, Goiânia - GO. Anais do VIII Workshop de Visão Computacional. 